\title{
Phase II trial of fenretinide in advanced renal carcinoma*
}

\author{
Ulka Vaishampayan $^{1}$, Lance K. Heilbrun ${ }^{2}$, Ralph E. Parchment ${ }^{1}$, Vikash Jain ${ }^{2}$, James Zwiebel ${ }^{3}$, \\ Ramesh R. Boinpally ${ }^{1}$, Patricia LoRusso ${ }^{1}$ and Maha Hussain ${ }^{4}$ \\ ${ }^{1}$ Division of Oncology and ${ }^{2}$ Biostatistics Core, Wayne State University/Karmanos Cancer Institute, Detroit, MI, USA; \\ ${ }^{3}$ National Cancer Institute, Bethesda, MD, USA $;{ }^{4}$ Departments of Internal Medicine and Urology, University of Michigan \\ Comprehensive Cancer Center, Ann Arbor, MI, USA
}

Key words: fenretinide, N-4-hydroxyphenyl retinamide, renal cancer, clinical trial

\begin{abstract}
Summary
Purpose: Fenretinide, a synthetic form of retinoid, induced apoptosis even in chemotherapy resistant cell lines. A phase II study was hence conducted to evaluate toxicity and efficacy of fenretinide in metastatic renal cancer. Methods: Eligibility included unresectable or metastatic renal cell carcinoma (RCC), adequate organ function and Zubrod performance status $\leq 2$. Prior immunotherapy and a maximum of one prior chemotherapy regimen were allowed. Fenretinide was administered at a dose of $900 \mathrm{mg} / \mathrm{m}^{2}$ twice daily orally for 7 days in a 21-day cycle. Toxicity was assessed at the start of each cycle, and response every 2 cycles. Results: Nineteen eligible patients enrolled of which fifteen had visceral/bone metastases. Seventeen patients had prior nephrectomy and 11 had prior immunotherapy. 76 cycles of therapy were delivered. Therapy was very well tolerated with few severe toxicities consisting of thrombosis in 1 individual and grade 3 fatigue, nausea and diarrhea in 1 patient. 5 patients had grade 2 nyctalopia and 3 patients had transient grade 2 visual toxicity. No objective responses were noted. Stable disease was seen in seven of nineteen cases $(37 \%, 90 \%$ C.I. 0.21-0.59). Median time to progression was 1.5 months and median duration of stable disease was 5.8 months (90\% C.I. 3.0-8.4). Median survival was 10 months. Tumor fenretinide levels were obtained in three patients and were in the lower end of the therapeutic range. Conclusion: Fenretinide was well tolerated but demonstrated minimal activity that was consistent with results of intratumoral drug measurements. Strategies are needed that will increase systemic and tumor levels of fenretinide.
\end{abstract}

\section{Introduction}

Retinoids are used in cancer therapy for their unique differentiation properties and their numerous roles in cellular function. All-trans retinoic acid is indicated in acute promyelocytic leukemia to differentiate blasts and facilitate the efficacy of chemotherapy [1]. Retinoids are vitamin A derivatives that exert a therapeutic effect in acute promyelocytic leukemia and preclinical antiproliferative effects in a variety of other malignancies such as breast, prostate and lung cancer [1, 2]. Experimental data in renal cancer cell lines suggested that 13-cis retinoic acid (13-CRA) augments the antiproliferative effect of interferon in interferon sensitive cell lines [3]. A phase II trial in advanced RCC with interferon and 13-CRA showed a $30 \%$ response rate and three complete responses. A randomized trial comparing 13-CRA and interferon with interferon alone demonstrated a higher

* Supported in part by NCI Cancer Center Support Grant CA-22453. response rate in the combination arm (12\% vs. $6 \%)$ but no difference in overall survival [4].

Fenretinide (NSC 374551, N-4-hydroxyphenyl retinamide, 4-HPR) is a synthetic retinoid that induces apoptosis and necrosis in tumor cells, including those that are unresponsive to retinoic acid [5]. Increase in reactive oxygen species mediating tumor cell death was also observed in retinoblastoma cell lines [6]. Clinically, fenretinide is better tolerated and long term administration is feasible [7]. Chemopreventive trials have been performed in breast and oral cancers at doses of $200 \mathrm{mg}$ daily $[8,9]$. A phase I study of fenretinide at our institution demonstrated the maximum tolerated dose of this agent as $1800 \mathrm{mg} / \mathrm{m}^{2}$ daily in two divided doses administered orally for 7 days in a 21-day cycle. One patient with metastatic renal carcinoma had an impressive clinical response. Based on the preclinical and clinical data of retinoids in renal carcinoma, and our experience with fenretinide, a phase II trial of this agent was conducted in metastatic renal cancer. 


\section{Patients and methods}

\section{Eligibility}

The eligibility criteria consisted of locally advanced unresectable, locally recurrent or metastatic RCC with no prior retinoid therapy. Treatment with biologic therapy and a maximum of one prior chemotherapy regimen were permitted. Concomitant administration of antioxidants was prohibited. All patients had to have unidimensionally measurable disease and pre study imaging for disease assessment within 28 days of registration. A Zubrod performance status of 0-2 was required. Patients had to have adequate bone marrow, liver (serum bilirubin, alkaline phosphatase and transaminases $\leq$ twice institutional upper limit of normal) and renal function (serum creatinine $\leq 2 \mathrm{mg} / \mathrm{dL}$ ). Prior radiation therapy was allowed, if completed at least four weeks prior to enrollment and only if there was measurable disease outside the radiation therapy port. Patients with brain metastases were excluded unless completely resected or irradiated resulting in a complete response. Individuals with a history of night blindness or retinal disease were excluded. No prior malignancy was allowed, except non-melanoma skin cancer, in-situ carcinoma of any site or other cancers for which the patient had been disease free for a minimum of two years. Pregnant and lactating females were excluded due to the possible teratogenic potential of the study medication. Patients in the reproductive age group had to practice effective contraception. All patients signed a written informed consent.

\section{Treatment plan}

The fenretinide was administered orally on an outpatient basis at a dose of $1800 \mathrm{mg} / \mathrm{m}^{2} /$ day rounded to the nearest $100 \mathrm{mg}$ in two divided doses daily for 7 days, every 21 days. The fenretinide was available as $100 \mathrm{mg}$ capsules and patients were instructed to take the medication with a fatty meal (usually whole milk) to improve the bioavailability. Compliance was monitored on the basis of calendars completed by patients. A dose decrease to $1400 \mathrm{mg} / \mathrm{m}^{2} /$ day was recommended if any toxicity grade 2 or higher occurred, and the next cycle of drug administration was restarted only after resolution of the toxicity to grade 1 or less.

\section{Measurement of fenretinide in tumor tissue}

\section{Collection of tumor biopsies}

On day 8 of the first cycle of therapy, tumor samples were collected via open biopsy (minimum size, $0.5 \times 0.5 \mathrm{~cm}$; maximum size, $1 \times 1 \mathrm{~cm}$ ) or core biopsy (minimum: 4 cores with 14 guage needle), as deemed appropriate by the surgeon. The biopsy was not mandatory and patients willing to undergo it signed a separate written informed consent. The tissue was placed into $1 \mathrm{cc}$ saline containing butylated hydroxytoluene (BHT), frozen on dry ice, and stored at $-80^{\circ} \mathrm{C}$ until HPLC analysis. The BHT stabilizes the fenretinide against oxidation during storage.

\section{Extraction of fenretinide from tumor tissue}

To a $0.5 \mathrm{~g}$ tumor biopsy specimen, an equal amount of refrigerated saline and $50 \mu \mathrm{L}$ of $40 \mathrm{mg} / \mathrm{mL}$ of BHT in acetonitrile were added and the specimen was homogenized. The homogenate was deproteinated by adding an equal volume of refrigerated acetonitrile and clarified by microcentriguation at $10,000 \mathrm{rpm}$ for $10 \mathrm{~min}$ at $4{ }^{\circ} \mathrm{C}$. A $150 \mu \mathrm{L}$ aliquot of supernatant was transferred into the small volume insert of an HPLC vial and $100 \mu \mathrm{L}$ injected into the HPLC.

\section{HPLC analysis}

A modified reversed phase liquid chromatographic (LC) method reported in the literature was used to quantify fenretinide [10]. Briefly, the method consisted of a Waters Resolve Spherical C-18 column, $150 \times 3.9 \mathrm{~mm}$ and $5 \mu \mathrm{m}$ particle size column fitted with a guard column (Waters Resolve Spherical C-18, $5 \mu \mathrm{m}$, Waters Corporation, USA). The mobile phase consisted of a mixture of acetonitrile: water: acetic acid (75:23:2), at a flow rate of $1 \mathrm{ml} / \mathrm{min}$. The eluent was monitored at $365 \mathrm{~nm}$ to detect the analyte. The concentration of analyte in tumor was determined by back calculation from a linear regression equation developed from external reference standards. Application of this method to measurement of fenretinide in homogenates of skin biopsies and of tumors from preclinical models has shown that fenretinide recovery is quantitative (data not shown). The LC method was reproducible, with low inter-day variability $(<4 \%)$, and the limit of quantitation under the described conditions was $50 \mathrm{ng}$ per $\mathrm{mL}$ of tumor homogenate.

\section{Response and toxicity evaluation}

Toxicity was assessed at the beginning of each cycle of therapy and response was evaluated every two cycles. The toxicities were graded as per the National Cancer Institute Common Toxicity Criteria, Version 2.0. The toxicities were recorded based on the greatest severity that occurred at any time during the treatment. The RECIST response criteria were utilized for assessment of response [11]. 


\section{Statistical methods}

This phase II trial was planned with a Simon optimal twostage design [12]. We wished to distinguish these regions of the true, unknown (complete + partial) response rate: 0.10 or less versus 0.30 or more. The 2 -stage design called for a maximum of 35 response-evaluable (r-e) patients, 18 in Stage 1 and 17 in Stage 2. The design had type I error of 0.05 and power of 0.90 . We needed at least 3 responders among the first $18 \mathrm{r}$-e patients to justify beginning Stage 2 . After 20 entries (over 17 months), we had 18 r-e patients. With no confirmed (partial) responders, the trial was terminated after the first stage was completed. Thus, after Stage 1, we concluded that the r-e sample response proportion $(0 / 18=0 \%)$ better supported the null hypothesis that the true, unknown response rate was at most 0.10 .

Exact, minimum-width $90 \%$ confidence intervals (CI) for response, stable disease, and toxicity rates were calculated using the Casella method [13], as implemented in StatXact software [14]. Standard Kaplan-Meier estimates of the censored overall survival (OS), time to progression (TTP), and stable disease duration (SDD) functions were computed. The toxicity evaluable population was the 19 patients who received any fenretinide treatment, whether eligible or not, to provide the most complete toxicity data from this trial. The time to event evaluable population was the 19 eligible patients, whether treated or not. Due to the small sample sizes, survival statistics (e.g., median) were estimated more conservatively using linear interpolation [15] among successive event times on the Kaplan-Meier curves. Duration of response or disease stabilization was measured from the start of therapy to progression or death. Overall survival was measured from the time of enrollment on the study to death or last follow-up.

\section{Results}

\section{Patient characteristics}

Twenty patients were enrolled on this study from December 2000 to May 2002. One patient was deemed ineligible after the pathology review indicated renal oncocytoma histology. The median age of the patients was 58 years (range 39-75 years). The patient characteristics are summarized in Table 1. 14 patients had clear cell histology, one patient had clear cell with sarcomatoid features and 4 patients had clear cell carcinoma with papillary features. 15 had visceral/bone metastases. 17 patients had prior nephrectomy and 11 had prior immunotherapy.

\section{Treatment summary}

A total of seventy-six cycles of therapy were administered. Median number of cycles received per patient were 2,

\begin{tabular}{|c|c|}
\hline Characteristic & No $(\%)$ \\
\hline \multicolumn{2}{|l|}{ Age } \\
\hline$\leq 60$ & $11(58)$ \\
\hline$>60$ & $8(42)$ \\
\hline \multicolumn{2}{|l|}{ Gender } \\
\hline Male & $12(63)$ \\
\hline Female & $7(37)$ \\
\hline \multicolumn{2}{|l|}{ Race } \\
\hline Caucasian & $15(78)$ \\
\hline Other & $2(11)$ \\
\hline African American & $2(11)$ \\
\hline \multicolumn{2}{|l|}{ Histology } \\
\hline Clear cell & $14(74)$ \\
\hline Papillary features & $4(21)$ \\
\hline Sarcomatoid features & $1(5)$ \\
\hline \multicolumn{2}{|l|}{ Performance status } \\
\hline 0 & $10(53)$ \\
\hline 1 & $8(42)$ \\
\hline 2 & $1(5)$ \\
\hline \multicolumn{2}{|l|}{ Surgery } \\
\hline Nephrectomy & $17(89)$ \\
\hline No nephrectomy & $2(11)$ \\
\hline \multicolumn{2}{|l|}{ Prior systemic treatment } \\
\hline None & $8(42)$ \\
\hline Immunotherapy & $7(37)$ \\
\hline Immunotherapy + chemo & $4(21)$ \\
\hline \multicolumn{2}{|l|}{ Metastatic sites } \\
\hline Visceral/Bone & $15(79)$ \\
\hline Lymph node/Soft Tissue & $4(21)$ \\
\hline
\end{tabular}

with a range of 1 to 12 cycles. 4 patients required dose reduction; 1 due to diarrhea and 3 due to ocular toxicity. No treatment related hospitalizations and mortalities were observed.

\section{Toxicity}

A comprehensive summary of toxicities is provided in Table 2. There were no grade 4 or 5 toxicities. Therapy was well tolerated with few severe toxicities consisting

Table 2. Toxicity profile

\begin{tabular}{lllll}
\hline Toxicity & Grade 1 & Grade 2 & Grade 3 & Grade 4 \\
\hline Nyctalopia & 0 & 5 & 0 & 0 \\
Anemia & 5 & 1 & 0 & 0 \\
Ocular & 1 & 7 & 0 & 0 \\
Photophobia & 1 & 1 & 0 & 0 \\
Fatigue & 5 & 3 & 1 & 0 \\
Dermatologic -rash & 0 & 2 & 0 & 0 \\
Nausea & 4 & 1 & 1 & 0 \\
Vomiting & 1 & 1 & 1 & 0 \\
Diarrhea & 3 & 1 & 1 & 0 \\
Lymphopenia & 2 & 1 & 0 & 0 \\
Creatinine & 1 & 2 & 0 & 0 \\
Thrombosis & 0 & 0 & 1 & 0 \\
\end{tabular}


of thrombosis in 1 patient, grade 2 anemia in 1 patient and grade 3 fatigue, diarrhea and nausea in 1 patient. Five patients had grade 2 nyctalopia and 3 patients had transient grade 2 visual toxicity. Four patients required dose reduction; 3 due to grade 2 nyctalopia and 1 due to grade 2 diarrhea.

\section{Response and survival}

Eighteen patients were evaluable for response as one eligible patient died of progressive disease before starting the fenretinide therapy. No objective responses were noted in the evaluable patients. Stabilization of disease (DS) was seen in 7 of 19 eligible patients $(37 \%$, 90\% C.I. 0.21-0.59). Of the 14 patients with documented disease progression prior to study entry, four had disease stabilization with therapy. Median time to progression was 1.5 months and median duration of DS was 5.8 months (90\% C.I. 3.0-8.4 months) (Figure 1). DS rates at 3 and 6 months were $71 \%$ and $38 \%$ respectively. The median survival in this pretreated advanced renal cancer population was 10 months (90\% C.I. 6.4-16.9 months) (Figure 2). The differences in survival and in progression-free survival by prior systemic therapy status were not statistically significant (Table 3).

\section{Correlative studies}

Four patients consented to the post therapy biopsy, and no severe complications related to the biopsy procedure were noted in these patients. HPLC analysis revealed detectable intratumoral levels of fenretinide in three biopsy specimens at $3.6 \mu \mathrm{M}(1.4 \mathrm{mcg} / \mathrm{g}), 3.8 \mu \mathrm{M}(1.5 \mathrm{mcg} / \mathrm{g})$ and $7.9 \mu \mathrm{M}(3.1 \mathrm{mcg} / \mathrm{g})$. In the remaining case, fenretinide was detected in the biopsy, but its level was below the limit of quantitation.

\section{Discussion}

Amongst the retinoids, 13-CRA was extensively evaluated in renal cancer and the initial clinical results were encouraging with a response rate of $30 \%$ in a phase II study in metastatic renal cancer [16]. In vitro studies demonstrated that 13-CRA potentiated the effects of interferon in interferon sensitive renal cancer cell lines [17]. Retinoic acid receptor (RAR) beta was induced in retinoid sensitive cell lines and probably mediated the anti-tumor efficacy of 13-CRA [15]. The results of the randomized clinical trial comparing interferon and 13-CRA [4] with interferon alone demonstrated higher response rate $(6 \%$ vs. $12 \%$ ) with the combination but no statistically significant

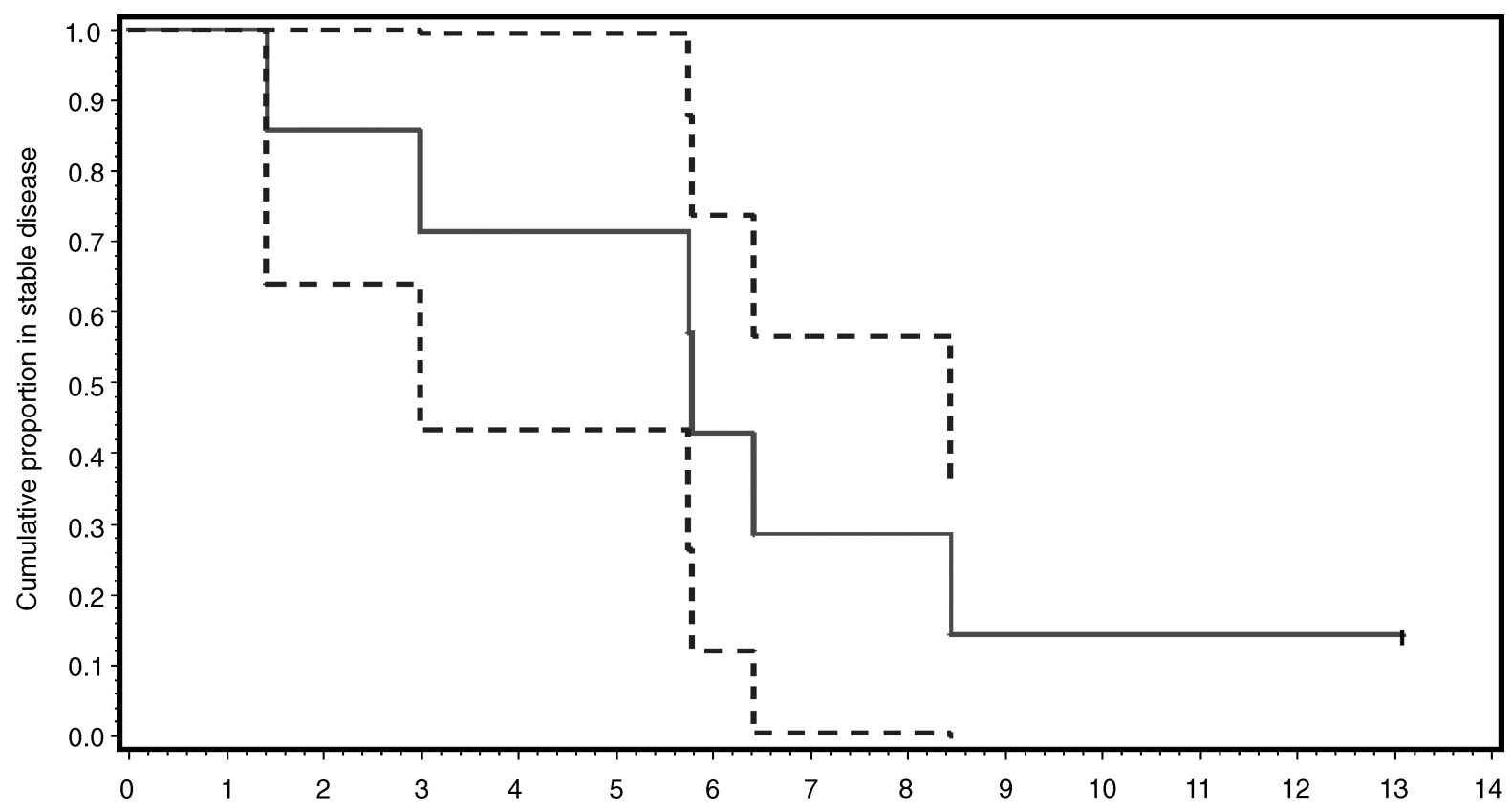

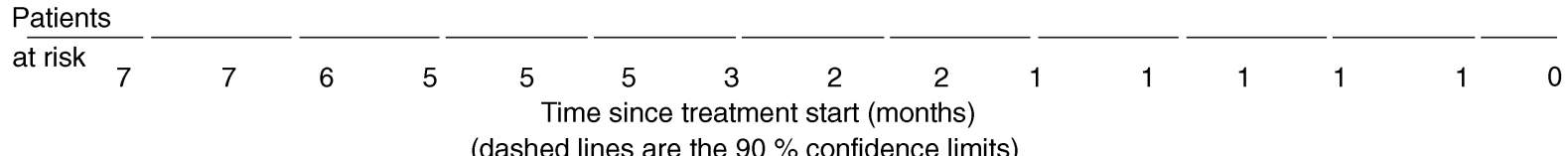

Figure 1. Kaplan-Meier estimate of stable disease duration. Dashed lines indicate $90 \%$ confidence limits. 


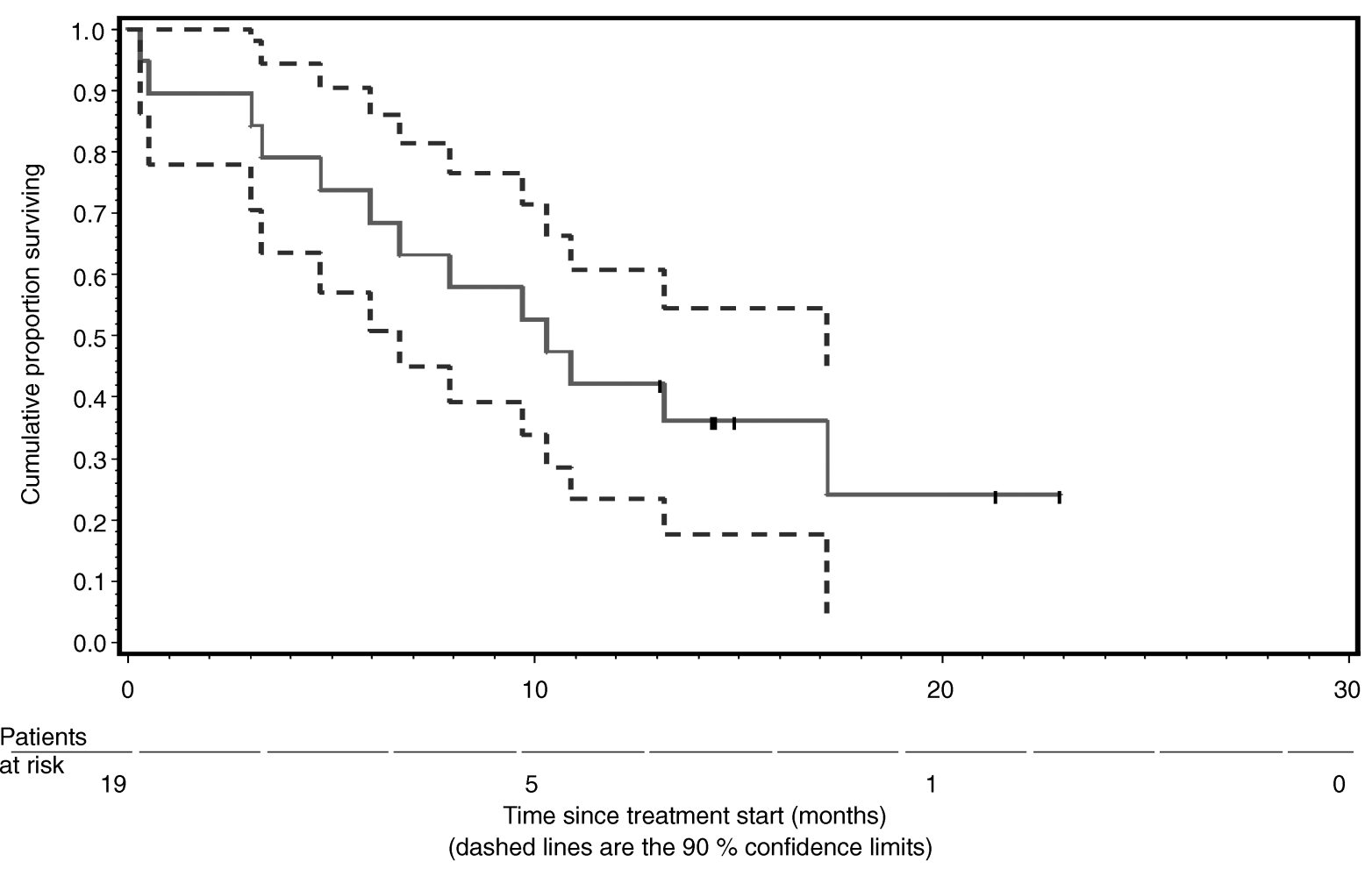

Figure 2. Kaplan-Meier estimate of overall survival of eligible patients. Dashed lines indicate $90 \%$ confidence limits.

difference in overall survival. However a higher proportion of patients appeared to have longer durations of response in 13-CRA arm. Hence a select population of renal cancers that have RAR-beta induction and interferon sensitivity will likely benefit from administration of retinoid therapy.

Fenretinide is a synthetic retinoid that emerged preclinically as a very active inducer of apoptosis independent of retinoic acid receptor alpha expression such as in HL-60R and NB-306 leukemia cell lines [17, 18]. At concentrations of 5-20 micromoles fenretinide demon-

Table 3. Overall survival and time to progression by prior systemic therapy status

\begin{tabular}{lll}
\hline & \multicolumn{2}{c}{ Any prior systemic therapy } \\
\cline { 2 - 3 } Endpoint & Yes $(n=11)$ & No $(n=8)$ \\
\hline Overall survival & & \\
$\quad$ Median (mo.) & $10.0(6.4,10.3)$ & $7.9(2.1, \mathrm{~N} / \mathrm{A})$ \\
6-month rate & $65 \%(0.42,0.89)$ & $62 \%(0.34,0.90)$ \\
$\quad$ 12-month rate & $34 \%(0.11,0.58)$ & $37 \%(0.08,0.67)$ \\
Time to progression & & \\
$\quad$ Median (mo.) & $1.4(1.3,1.8)$ & $1.8(0.8,5.9)$ \\
3-month rate & $25 \%(0.03,0.46)$ & $25 \%(0.00,0.50)$ \\
6-month rate & $8 \%(0.00,0.21)$ & $14 \%(0.00,0.34)$ \\
\hline
\end{tabular}

Table entries are the point estimate of the survival statistic, with its $90 \%$ confidence limits shown underneath, in parentheses.

$\mathrm{N} / \mathrm{A}=$ not available, due to censoring pattern and small sample size. strated potent activation of retinoic acid receptor gamma and moderate activation of the retinoic acid beta receptors. Multiple other mechanisms of antiproliferative effects of fenretinide have been described including generation of free oxygen radicals and down regulation of c-myc, cyclin D1 and p34 leading to apoptotic effect [17-20].

Clinical investigation of fenretinide has primarily been conducted in breast cancer with prolonged administration of lower doses for chemoprevention [21]. A large study in 54 children with neuroblastoma revealed that dose escalation upto $4000 \mathrm{mg} / \mathrm{m}^{2}$ for 28 days administration was feasible [22]. There was about a 10 -fold difference in the peak plasma levels with dose escalation from $100 \mathrm{mg} / \mathrm{m}^{2} /$ day to $4000 \mathrm{mg} / \mathrm{m}^{2}$ daily. The 28 -day administration caused approximately 2 -fold increase in the levels for each dose when compared with the levels after 7 days administration (from 0.6 to $1.3 \mu \mathrm{M}$ and from 6 to $12.9 \mu \mathrm{M}$ ). These plasma concentrations have been shown to induce apoptosis in vitro in neuroblastoma cell lines.

However, the relationship between plasma and tissue levels of fenretinide is poorly understood, so it is not clear if achieving in vitro-active concentrations in the plasma will result in similarly high drug exposure in tumor tissue. To that end, our study provided an opportunity to examine accumulated intratumoral levels of fenretinide by obtaining tumor biopsies after seven days of twice daily administration. In the current study of the $1800 \mathrm{mg} / \mathrm{m}^{2}$ daily for 
seven days regimen, tumor fenretinide levels measured $12-22 \mathrm{~h}$ after the last dose ranged from 3.6 to $7.9 \mu \mathrm{M}$, which approximated levels expected in the tumor after one half-life (plasma half-life in Phase I patients was 8-14 h, data not shown), assuming that drug freely distributes between plasma and tumor. However, the in vitro assays of solid tumor lines [24-26], including neuroblastoma lines [27], also reveal a requirement to maintain fenretinide concentrations at $10 \mu \mathrm{M}$ for a minimum of $72 \mathrm{~h}$ to elicit 50-90\% growth inhibition and apoptosis. Taken together, these results indicate that the effective in vitro concentration range of fenretinide in other human solid tumor cell lines was reached in the renal tumors treated in our study, sometime between third and fourth day of therapy and intratumoral levels were fluctuating between effective and ineffective concentrations for the remainder of the treatment period. To our knowledge there are no reports of optimal therapeutic concentrations of fenretinide levels in renal cancer cell lines. Hence it is unknown whether the fenretinide levels found to be therapeutic in lung, breast and cervix cancer cell lines would also be effective in renal cancer. The levels that have been found to be sub therapeutic in other cancers are suggestive of a reason for the lack of any objective responses in the study in advanced renal cancer.

In conclusion, fenretinide demonstrated disease stabilization in seven patients, of which three had stable disease lasting for at least 6 months. The study also indicates that prolonged administration at higher doses is feasible and well tolerated, and could be considered in combination with other effective therapies. However, the data clearly show that fenretinide as a single agent has unremarkable activity in RCC, so any further clinical development of this drug for RCC would require a strong scientific rationale and preferably also an improved dosage form that will maintain intratumoral levels above $10 \mu \mathrm{M}$ for 72 consecutive hours or longer.

\section{Acknowledgments}

We extend our thanks to the Investigational Drug Branch of the National Cancer Institute for providing the fenretinide used in this study and to Sen-Lin Zhou for providing technical support. This work was supported in part by grant UO1-CA-062487 from the National Cancer Institute and by NIH Cancer Center Support Grant CA-22453.

\section{References}

1. Tallman MS, Andersen JW, Schiffer CA, Appelbaum FR, Feusner JH, Ogden A, Shepherd L, Willman C, Bloomfield CD, Rowe JM et al.: All-trans-retinoic acid in acute promyelocytic leukemia. $\mathrm{N}$ Engl J Med 337: 1021-1028, 1997
2. Camacho LH: Clinical applications of retinoids in cancer medicine. J Biol Regul Homeost Agents. Jan-Mar 17: 98-114, 2003

3. Taguchi I, Hara I, Gohji K, Arakawa S, Kamidono S: Synergistic anti-tumor effect of 13-cRA and IFN-alpha/beta in mouse renal cell carcinoma. Int J Oncol 13: 145-149, 1998

4. Motzer RJ, Murphy BA, Bacik J, Schwartz LH, Nanus DM, Mariani T, Loehrer P, Wilding G, Fairclough DL, Cella D et al.: Phase III trial of interferon alfa-2a with or without 13-cis-retinoic acid for patients with advanced renal cell carcinoma. J Clin Oncol 18: $2972-$ 2980, 2000

5. Delia D, Aiello A, Lombardi L, Pelicci PG, Grignani F, Grignani F, Formelli F, Menard S, Costa A, Veronesi U et al.: $\mathrm{N}$-(4-hydroxyphenyl)retinamide induces apoptosis of malignant hemopoietic cell lines including those unresponsive to retinoic acid. Cancer Res 53: 6036-6041, 1993

6. Tosetti F, Vene R, Arena G, Morini M, Minghelli S, Noonan DM, Albini A: N-(4-hydroxyphenyl)retinamide inhibits retinoblastoma growth through reactive oxygen species-mediated cell death. Mol Pharmacol 63: 565-573, 2003

7. Costa A, Malone W, Perloff M, Buranelli F, Campa T, Dossena G, Magni A, Pizzichetta M, Andreoli C, Del Vecchio M et al.: Tolerability of the synthetic retinoid Fenretinide (HPR). Eur J Cancer Clin Oncol 25: 805-808, 1989

8. Torrisi R, Decensi A, Formelli F, Camerini T, De Palo G: Chemoprevention of breast cancer with fenretinide. Drugs 61: 909-918, 2001

9. Chiesa F, Tradati N, Marazza M, Rossi N, Boracchi P, Mariani L, Fornelli F, giardini R, Costa A, DePalo G et al.: Fenretinide (4-HPR) in chemoprevention of oral leukoplakia. J Cell Biochem Suppl 17F: 255-261, 1993

10. Formelli F, Carsana R, Costa A, Buranelli F, Campa T, Dossena G, Magni A, Pizzichetta M: Plasma retinol level reduction by the synthetic retinoid fenretinide: A one year follow-up study of breast cancer patients. Cancer Res 49: 6149-1652, 1989

11. Therasse P, Arbuck SG, Eisenhauer EA, Wanders J, Kaplan RS, Rubinstein, L, Verweij, J, Van Glabbeke, M, van Oosterom, AT, Christian MC et al.: New guidelines to evaluate the response to treatment in solid tumors. J Natl Cancer Inst 92: 205-216, 2000

12. Fleming TR: One-sample multiple testing procedure for Phase II clinical trials. Biometrics 38: 143-151, 1982

13. Casella G: Refining binomial confidence intervals. Canadian J Statistics 14: 113-129, 1987

14. Mehta C, Patel N: StatXact 5: Statistical Software for Exact Nonparametric Inference, User Manual. Cytel Software Corporation, Cambridge, MA 1999, pp. 429-433

15. Lee ET: Statistical Methods for Survival Data Analysis, 2nd Edition Wiley \& Sons, Inc, New York, 1992, pp. 77-78

16. Motzer RJ, Schwartz L, Law TM, Murphy BA, Hoffman AD, Albino AP, Vlamis V, Nanus DM: Interferon alfa-2a and 13-cis-retinoic acid in renal cell carcinoma: Antitumor activity in a phase II trial and interactions in vitro. J Clin Oncol 13: 1950-1907, 1995

17. Nanus DM, Geng Y, Shen R, Lai HK, Pfeffer SR and Pfeffer LM Interaction of retinoic acid, interferon in renal cancer cell lines. $\mathrm{J}$ Interferon Cytokine Res 20: 787-794, 2000

18. Delia D, Aiello A, Formelli F, Fontanella E, Costa A, Miyashita T, Reed JC, Pierotti MA: Regulation of apoptosis induced by the retinoid $\mathrm{N}$-(4-hydroxyphenyl) retinamide and effect of deregulated bcl-2. Blood 85: 359-367, 1995

19. Sun SY, Li W, Yue P, Lippman SM, Hong WK, Lotan R: Mediation of N-(4-hydoxyphenyl)retinamide-induced apoptosis in human cancer cells by different mechanisms. Cancer Res 59: 2493-2498, 1999

20. Dipietrantonio A, Hsieh TC, Wu JM: Differential effects of retinoic acid (RA) and N-(4-hydroxyphenyl) retinamide (4-HPR) on cell growth, induction of differentiation, and changes in $\mathrm{p} 34 \mathrm{cdc} 2$, Bcl-2 and actin expression in the human promyelocytic HL-60 
leukemic cells. Biochem Biophys Res Commun 224: 837-842, 1996

21. Fontana JA, Rishi AK: Classical and novel retinoids: Their targets in cancer therapy. Leukemia 16: 463-472, 2002

22. Camerini T, Mariani L, De Palo G, Marubini E, Di Mauro MG, Decensi A, Costa A, Veronesi U: Safety of the synthetic retinoid fenretinide: Long-term results from a controlled clinical trial for the prevention of contralateral breast cancer. J Clin Oncol 19: 16641670, 2001

23. Garaventa A, Luksch R, Piccolo MS, Cavadini E, Montaldo PG, Pizzitola MR, Boni L, Ponzoni M, Decensi A, Bernardi BD, Bellani FF, Formelli F: Phase I trial and pharmacokinetics of fenretinide in children with neuroblastoma. Clin Cancer Res 9: 2032-2039, 2003

24. Kalemkerian GP, Slusher R, Ramalingam S, Gadgeel S, Mabry M: Growth inhibition and induction of apoptosis by fenretinide in small-cell lung cancer cell lines. J Natl Cancer Inst 87: 16741680, 1995
25. Marth C, Bock G, Daxenbichler G: Effect of 4hydroxyphenylretinamide and retinoic acid on proliferation and cell cycle of cultured human breast cancer cells. J Natl Cancer Inst 75: 871-875, 1985

26. Oridate N, Lotan D, Mitchell MF, Hong WK, Lotan R: Inhibition of proliferation and induction of apoptosis in cervical carcinoma cells by retinoids: Implications for chemoprevention. J Cell Biochem Suppl 23: 80-86, 1995

27. Mariotti A, Marcora E, Bunone G, Costa A, Veronesi U, Pierotti MA, Della Valle G: N-(4-hydroxyphenyl)retinamide: A potent inducer of apoptosis in human neuroblastoma cells. J Natl Cancer Inst 86: $1245-1247,1994$

Address for offprints: Ulka Vaishampayan M.D., Karmanos Cancer Institute, 4th Floor Hudson-Webber Cancer Research Center, 4100 John R Road, Detroit, MI 48201, USA. Tel.: 313-745-8296; Fax: 313-993-0559;

E-mail: vaishamu@karmanos.org 